\title{
Interlink between Tunable Material Properties and Thermoresponsiveness of Cross-Linked Poly(N-vinylcaprolactam) Thin Films Deposited by Initiated Chemical Vapor Deposition
}

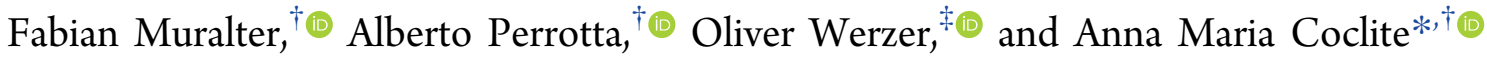 \\ ${ }^{\dagger}$ Institute of Solid State Physics, NAWI Graz, Graz University of Technology, Petersgasse 16, 8010 Graz, Austria \\ ${ }^{\ddagger}$ Institute of Pharmaceutical Science, Department of Pharmaceutical Technology, University of Graz, Universitaetsplatz 1, 8010 \\ Graz, Austria
}

Supporting Information

ABSTRACT: In this contribution, we report on the thin-film synthesis of a novel thermoresponsive polymer, namely, poly $(N$-vinylcaprolactam $)$ cross-linked by di(ethylene glycol)divinyl ether [p(NVCL-co-DEGDVE)] by initiated chemical vapor deposition (iCVD). Its transition between swollen and shrunken states in film thickness and the corresponding lower critical solution temperature (LCST) was investigated by spectroscopic ellipsometry in water. Water contact angle measurements and nano-indentation experiments reveal that the transition is accompanied by a change in wettability and elastic modulus. The amount of cross-linking was used to tune the thermoresponsive behavior of the thin films, resulting in higher swelling and LCST, increased surface rearrangement, and lower stiffness for less cross-linked polymers. For the first time, the filament temperature during iCVD synthesis was used to vary the chain length of the resulting polymeric systems and, thus, the position of their thermoresponsive transition. With that, swelling of up to $250 \%$ compared to the dry thickness and transition temperatures ranging from 16 to $40{ }^{\circ} \mathrm{C}$ could be achieved.

\section{INTRODUCTION}

Smart hydrogel materials can be used in a variety of sensor ${ }^{1}$ and actuator $^{2}$ setups because of their ability to react to a number of external stimuli including temperature, $\mathrm{pH}$, magnetic/electric fields, or concentrations of specific chemical species (e.g., glucose). ${ }^{3}$ In an aqueous environment, such a hydrogel binds water molecules because of its network of hydrophilic polymer chains, making the material rearrange and swell to multiples of its dry size. Upon changing the environment, the smart polymer reversibly takes up water into or repels out water from its inherent structure.

Kinetically, the water exchange is time-limited by water diffusivity. ${ }^{4}$ Thus, fast response times and, in turn, optimal device performance, can be achieved by employing thin polymeric films. However, the water exchange imposes stress on the film and could eventuate in delamination or mechanical failure. To ensure mechanical stability, a cross-linking agent can be co-polymerized with the monomer exhibiting the initially desired functionalities. The cross-linked hydrogel can be viewed as a polymer mesh (with a certain mesh size), which can take up a specific amount of water into its structure. ${ }^{5}$

The devices, where smart polymer thin films are usually applied, involve delicate surfaces (e.g., drugs and flexible substrates), oftentimes exhibiting sophisticated nanostructure, which need to be coated conformally. For these reasons, instead of ordinary solution processing, in this contribution,

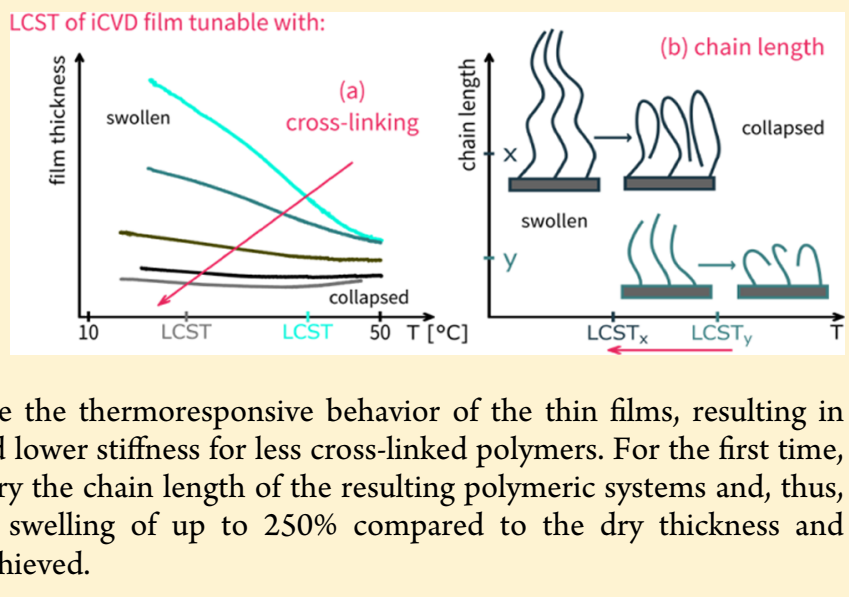

initiated chemical vapor deposition (iCVD) was used. With this solvent-free technique, functional co-polymers of desired composition can be vapor-phase-synthesized similar to free radical polymerization processes. ${ }^{6}$ The low substrate temperature employed $\left(10-40{ }^{\circ} \mathrm{C}\right)$ allows for the coating also of delicate substrates. In these mild processing conditions, the full retention of delicate functional groups such as the adopted thermoresponsive species can be achieved upon deposition.

The chemical nature of monomer and cross-linker used in iCVD allows the further tailoring of the response behavior of the resulting smart polymer. For example, the most prominent thermoresponsive hydrogel, namely, poly- $N$-isopropylacrylamide (pNIPAAm), exhibits hydrophilic groups (i.e., amide) that are able to bind water and also the effective intrachain interactions causing the polymer to collapse. ${ }^{7}$ The predominant behavior depends on temperature with the transition point being the lower critical solution temperature (LCST). Therefore, if in aqueous environment, the polymer accordingly undergoes a phase transition from a hydrated swollen state below to a dehydrated shrunken state above the LCST. Other thermoresponsive polymers with different functional groups interact differently with water and, therefore, vary in

Received: July 1, 2019

Revised: August 12, 2019

Published: August 30, 2019 
thermoresponsive behavior compared to pNIPAAm. For example, poly $(N, N$-diethylacrylamide) (pDEAAm) hydrogels show a broader LCST transition in water compared to pNIPAAm hydrogels. ${ }^{8}$ In humidity, a similar trend has been reported for pDEAAm-based thin films deposited by iCVD.? Besides that, cross-linking has been reported to affect the thermoresponsiveness and, thus, where the LCST transition takes place. More hydrophobic ${ }^{10}$ or hydrophilic ${ }^{11}$ cross-linking agents can be used to deliberately control the direction of the shift.

As polymer synthesis is concerned, the NIPAAm monomer is solid at room temperature and exhibits low vapor pressure even at elevated temperatures (e.g., $85^{\circ} \mathrm{C}$ ); hence, different thermoresponsive agents are sought allowing for easier handling and, hence, enabling to exploit the full spectrum of possibilities in thin-film deposition by iCVD. Lee et al. reported on synthesizing another thermoresponsive polymer, namely, poly $(N$-vinylcaprolactam) (pNVCL), for the first time by iCVD. ${ }^{12}$ This polymer is known for its nontoxicity and biocompatibility. ${ }^{13}$ In contrast to, for example, pNIPAAm, its specific chemical structure (with the caprolactam ring) results in a "classical" Flory-Huggins miscibility behavior, for which increasing the polymer chain length leads to a downward shift of the critical point (LCST). ${ }^{13,14}$ For different molecular weight samples, transition temperatures in the range of $\sim 30-$ $40{ }^{\circ} \mathrm{C}$ have been reported. ${ }^{14}$ Lee et al.reported on the synthesis of pNVCL homopolymer thin films by iCVD. ${ }^{12}$ Pretreatment of the substrates by an oxygen plasma was used for grafting the polymer onto the surface. ${ }^{2}$ In the present contribution, we aim at circumventing this step by introducing a cross-linking agent that allows for stabilization of the thermoresponsive polymer layer on the substrate without further synthesis efforts. Moreover, the cross-linker used, namely, di(ethylene glycol) divinyl ether (DEGDVE), has been previously shown to allow for tuning of the thermoresponsiveness in pNIPAAm-based copolymers by our group. ${ }^{9,15}$ Thus, the amount of DEGDVE will be utilized to tailor the response also of this novel co-polymer. To further tune the response properties of the deposited systems, we seek to employ the filament temperature during iCVD synthesis to control the molecular weight of the resulting polymers. We aim at demonstrating that differences in molecular weight will eventuate in differences in the position of the LCST transition for the first time.

\section{EXPERIMENTAL SECTION}

Thin-Film Synthesis. pNVCL-based thin films were deposited in a custom-built iCVD reactor. The experimental setup has already been described elsewhere. ${ }^{15}$ In the present contribution, single-sided polished silicon wafer substrates with a native oxide of $1.6 \mathrm{~nm}$ thickness on top (Siegert Wafer, Germany) were used as substrates. All the depositions were run at substrate temperatures of $35^{\circ} \mathrm{C}$ at a working pressure of $250 \mathrm{mT}$ Torr. tert-Butyl peroxide (TBPO, 98\%; Aldrich, Germany), kept at room temperature, was used as the initiator at a flow rate of $1 \mathrm{sccm}$. The filament temperature was used to control the number of initiator radicals formed, as described in more detail later. N-Vinylcaprolactam (NVCL, 98\%; Aldrich, Germany) is used as monomer and DEGDVE (99\%; Aldrich, Germany) as cross-linker. To achieve the desired flow rates, NVCL and DEGDVE are kept at elevated temperatures of 78 and $70^{\circ} \mathrm{C}$, respectively. The monomer flow rate is set to $0.2 \mathrm{sccm}$. A series of films was deposited at a filament temperature of $200{ }^{\circ} \mathrm{C}$ with varying amounts of cross-linking in consequence of changing the cross-linker flow rate in the range of $0-2 \mathrm{sccm}$. Another series of samples with constant nominal cross-linking of $20 \%$ was deposited at varied filament temperatures between 165 and $215{ }^{\circ} \mathrm{C}$, with and without nitrogen as patch flow; $\mathrm{N}_{2}$ does not participate to the chemical reactions but allows for a better gas/vapor flow distribution in the reactor and was used at a flow rate of $2 \mathrm{sccm}$. For this series, the monomer and cross-linker flow rates were both kept constant at 0.2 $\mathrm{sccm}$. All the corresponding $p_{\mathrm{M}} / p_{\text {sat }}$ values (ratios of the vapor pressure of the monomer/cross-linker to the saturation vapor pressure) are below 0.2, where a linear relation to the surface concentration of the chemical species has been reported for iCVD. ${ }^{16}$

In the present work, all the investigated $\mathrm{p}$ (NVCL-co-DEGDVE) thin films were deposited with similar film thickness values of $(50 \pm$ 5) $\mathrm{nm}$. The series of thin films prepared with varying amounts of cross-linking was deposited with average deposition rates of around 2 $\mathrm{nm} / \mathrm{min}$, whereas the filament temperature series varied in terms of average deposition rate between 0.1 and $2 \mathrm{~nm} / \mathrm{min}$, as described in more detail later.

Thin Film Characterization. Infrared absorbance spectra of the thin-film samples (1000 scans) were collected in transmission mode on a MB-102 (Bomem, Canada) Fourier transform infrared (FTIR) spectrometer in the wavenumber range $400-4000 \mathrm{~cm}^{-1}$ at a resolution of $4 \mathrm{~cm}^{-1}$ in mid IR mode.

Spectroscopic ellipsometry (SE) in a wavelength range of 370$1000 \mathrm{~nm}$ (M-2000S, J.A. Woollam, USA) was applied to determine film thickness and optical properties of the thin films in various environments (room temperature in nitrogen atmosphere or heating ramps in water). The swelling experiments in deionized water were performed in a temperature-controlled liquid stage (J. A. Woollam, USA). The recorded data was evaluated with an optical model consisting of a $\mathrm{c}-\mathrm{Si}$ semi-infinite layer on the bottom (temperature dependent), a $1.6 \mathrm{~nm}$ thick native $\mathrm{SiO}_{2}$ layer in the middle and the polymer film on top, modeled as a Cauchy function with an Urbach tail accounting for adsorption in the low-wavelength region. $\mathrm{H}_{2} \mathrm{O}$ with temperature-dependent optical properties was set as the surrounding medium. For the temperature-dependent swelling experiments, the liquid stage and the mounted sample (already exposed to deionized water) were precooled to $\sim 15{ }^{\circ} \mathrm{C}$. The respective signal was then recorded while applying a temperature ramp from 15 to $50{ }^{\circ} \mathrm{C}$ at a heating rate of $0.5^{\circ} \mathrm{C} / \mathrm{min}$. Directly after deposition, the thin-film samples were rinsed for $30 \mathrm{~s}$ with deionized water for equilibration. For thermal equilibration reasons reported earlier, ${ }^{15,17}$ the temperature-dependent swelling behavior and the LCST of the ICVD thin films were evaluated from the third heating experiment. The SE measurements in dry $\mathrm{N}_{2}$ atmosphere were performed in a THMS600 temperature stage (Linkam, UK) at room temperature $\left(\sim 25^{\circ} \mathrm{C}\right)$. The samples were measured after $30 \mathrm{~min}$ of nitrogen flow, so that the film thickness would not vary for more than $0.5 \mathrm{~nm}$ in $5 \mathrm{~min}$. The same optical model as in the liquid case has been used to evaluate the recorded data, but with the ambient material being set to air $(n \approx 1)$.

Water contact angle (WCA) measurements were performed on a CAM 200 optical contact meter (KSV Instruments Ltd., Finland) equipped with a THMS600 temperature stage (Linkam, UK). For each measurement, the sample temperature was set to the desired value, measured with a thermocouple on the surface of the sample and equilibrated for $5 \mathrm{~min}$ before the actual water drop was applied. Advancing and receding contact angles were measured via the volume changing method. Thus, 5 drops were used on each sample and 15 advancing and 5 receding contact angles were evaluated for each sample.

For the mechanical properties of the swollen films, nanoindentation measurements were performed using an atomic force microscope (FlexAFM with a C3000 controller from Nanosurf, Switzerland). A contact mode ContAl-G cantilever (Budgetsensor, Bulgaria) with a nominal tip radius of $10 \mathrm{~nm}$ was used. The spring constant was determined using the Sader thermal vibration approach. ${ }^{18}$ The samples were measured using deionized water. The temperature was controlled by a bioheater (Nanosurf, Switzerland) with a control unit of Warner Instruments Corporation (Hamden, CT, USA). Deflection-separation curves were recalculated to forceversus-separation curves using MATLAB scripts employing the approach described elsewhere. ${ }^{19}$ The apparent elastic moduli were derived and evaluated via the AtomicJ software package from 16 force 
curves measured on each sample. ${ }^{20}$ The individual measurements were taken at different positions of $1 \mu \mathrm{m}$ lateral separation.

\section{RESULTS AND DISCUSSION}

Chemical Composition. For the first time, p(NVCL-coDEGDVE) thin films with varying amounts of DEGDVE crosslinker content have been successfully polymerized by iCVD. Different degrees of cross-linking have been achieved by changing the cross-linker flowrate during deposition. The $p_{\mathrm{M}} /$ $p_{\text {sat }}$ values in the applied temperature and pressure conditions have been used to precalculate the composition of the films to be deposited (i.e., nominal cross-linking degree) with the following formula

$$
\begin{aligned}
& \text { DEGDVE fraction [\%] } \\
& =\frac{\left(p_{\mathrm{M}} / p_{\mathrm{sat}}\right)_{\mathrm{DEGDVE}}}{\left(p_{\mathrm{M}} / p_{\mathrm{sat}}\right)_{\mathrm{NVCL}}+\left(p_{\mathrm{M}} / p_{\mathrm{sat}}\right)_{\mathrm{DEGDVE}}}
\end{aligned}
$$

The nominal DEGDVE fraction has been varied between 0 and $75 \%$. As reported also in the literature, ${ }^{21}$ such nominal cross-linker fraction does not always correspond to the real cross-linking fraction in the polymer. This is attributable to the different reaction rates of the polymerizable vinyl groups in the monomer and in the cross-linker. In particular, in this case, DEGDVE does not self-polymerize. Considering that it can only directly react with NVCL, it can be assumed that the maximum amount of incorporable DEGDVE should be around 30 and not $75 \%$. Thus, a large quantity of DEGDVE adsorbed on the surface remains unreacted. While this behavior might be undesired, the dependency between nominal and real DEGDVE fraction is monotone; a higher nominal amount present on the surface during polymerization also gives a higher real amount of DEGDVE incorporated into the synthesized polymer, as confirmed by the experiments shown later.

To gain information about the chemical composition, the deposited films were investigated by FTIR absorbance spectroscopy. The FTIR data confirm the successful polymerization by all the recorded spectra not exhibiting characteristic vinyl group vibrations at 3150,1620 , and $1400 \mathrm{~cm}^{-1}$ as labeled by Salzmann et al. ${ }^{9}$ A representative spectrum of a nominally $75 \%$ cross-linked $p$ (NVCL-co-DEGDVE) thin-film sample is shown in Figure 1. The recorded absorption bands compare well to FTIR data of homopolymeric pNVCL films deposited by iCVD in the literature, ${ }^{12}$ with all recorded peaks being successfully assigned to absorption bands within the chemical structure of pNVCL according to Lee et al. ${ }^{12}$ The literature shows that DEGDVE is lacking strong characteristic FTIR absorption bands; ${ }^{15,22}$ therefore, it is not possible to evaluate the cross-linker amounts from the FTIR data collected on nominally differently cross-linked samples.

Behavior in Water. Upon rinsing, samples exhibiting nominal degrees of cross-linking below $10 \%$ appear to swell (e.g., change in color) but are rather unstable in water and even delaminate from the substrate suggesting that an insufficient co-polymerization has taken place. In turn, the fact that stable films result for nominal cross-linking equal to or above $10 \%$ indicates that a successful co-polymerization of the monomer NVCL with the cross-linker DEGDVE was achieved. These samples allow then the swelling behavior to be investigated in more detail. Results of the temperaturedependent swelling experiments in water recorded by SE on the differently cross-linked polymers are shown in Figure 2.

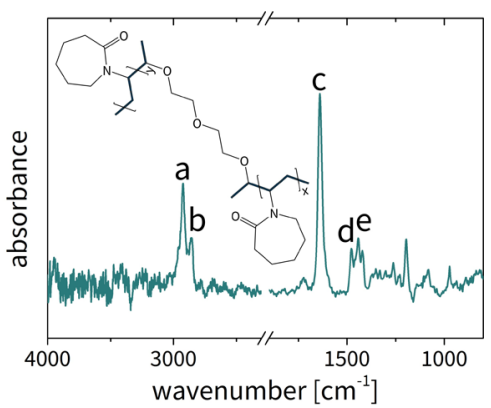

\begin{tabular}{llr} 
Label & Assignment & Position $\left[\mathrm{cm}^{-1}\right]$ \\
\hline a & aliphatic C-H stretch (in ring) & 2920 \\
b & amine III (C-N) & 2850 \\
C & C=O stretch & 1640 \\
d & amide (C-N) & 1480 \\
e & aliphatic C-C (in ring) & 1440
\end{tabular}

Figure 1. FTIR absorption spectrum of a nominally $75 \%$ cross-linked $\mathrm{p}$ (NVCL-co-DEGDVE) $50 \mathrm{~nm}$ thin-film sample to indicate successful polymerization with the absence of characteristic vinyl group vibrations at 3150,1620 , and $1400 \mathrm{~cm}^{-1}$ as labeled by Salzmann et al.; ${ }^{9}$ peaks of pNVCL assigned as labeled by Lee et al. ${ }^{12}$

The swelling behavior changes with the cross-linker content. This tunability further proves the successful co-polymerization of NVCL and DEGDVE at different ratios.

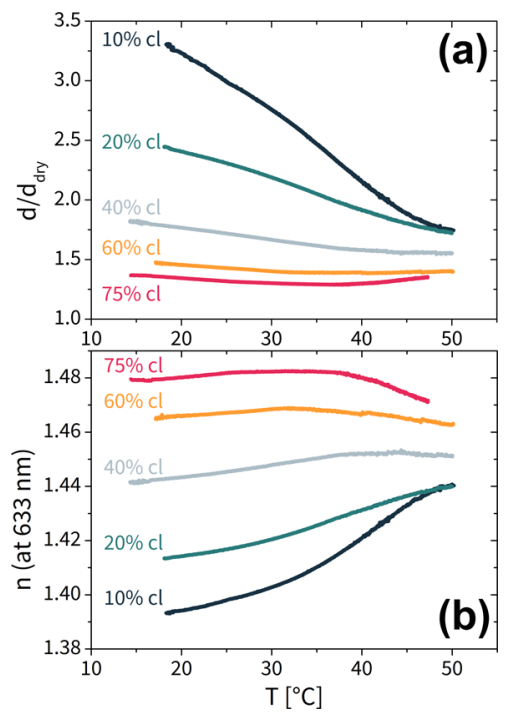

Figure 2. Thickness of the swollen layers normalized by the dry thickness $d / d_{\text {dry }}$ (a) and refractive index $n$ (b) during swelling in water recorded by $\mathrm{SE}$ while applying heating ramps from $\sim 15$ to $50{ }^{\circ} \mathrm{C}$ for various differently cross-linked $\mathrm{p}$ (NVCL-co-DEGDVE) thin-film samples.

All samples presented in Figure 2 show thermoresponsive swelling behavior by changing their film thickness and refractive index upon heating. The LCST values of the differently cross-linked $\mathrm{p}$ (NVCL-co-DEGDVE) samples have been evaluated as the mean values of the points of inflection of the respective swelling curves (exemplary data is shown in the Supporting Information). The LCST values are plotted in Figure $3 \mathrm{a}$ as a function of the DEGDVE content. They vary from 40 to $23{ }^{\circ} \mathrm{C}$. In the literature, an LCST of $31{ }^{\circ} \mathrm{C}$ was reported for a grafted $\mathrm{pNVCL}$ homopolymer thin film deposited by iCVD, ${ }^{12}$ while we measure an LCST of $40{ }^{\circ} \mathrm{C}$ for the least cross-linked sample. We think such difference can 


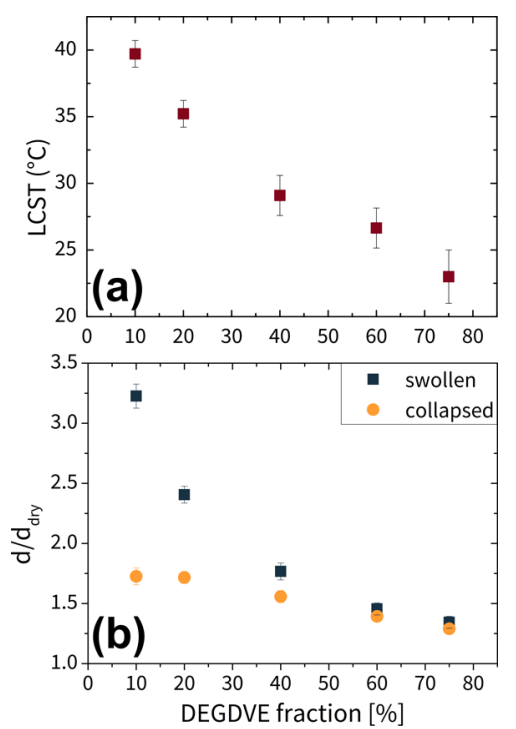

Figure 3. (a) LCST and (b) thickness normalized by the dry thickness $d / d_{\text {dry }}$ in the swollen $\left(@ 20^{\circ} \mathrm{C}\right)$ and in the collapsed state as a function of the nominal DEGDVE fraction of various differently cross-linked $\mathrm{p}$ (NVCL-co-DEGDVE) thin films.

be explained, hypothesizing that our samples exhibit lower molecular weight because they were deposited also at lower $p_{\mathrm{M}} / p_{\text {sat }}$. The $p_{\mathrm{M}} / p_{\text {sat }}$ values employed during deposition and, as a consequence, the deposition rate, are known to influence the molecular weight of iCVD thin films, with increasing deposition rate yielding higher molecular weight polymers. $^{23}$ The LCST of pNVCL in water has been shown to strongly depend on the molecular weight (i.e., chain length). ${ }^{14}$ Meeussen et al.report on an increase of the viscosity-average molecular weight from 9 to 20 to $275 \mathrm{~kg} / \mathrm{mol}$ lowering the transition temperature from 38 to 35 to below $30{ }^{\circ} \mathrm{C}$, respectively. $^{14}$

Moreover, the results in Figure 3a also show that the LCST decreases with adding DEGDVE to the polymeric structure, from 40 to $23{ }^{\circ} \mathrm{C}$. This is in agreement with our previous work on DEGDVE, showing that the LCST of pNIPAAm-based thin films synthesized by iCVD also decreases with their DEGDVE content to a similar extent. ${ }^{15}$ The hydrophobicity of the crosslinker has been deemed responsible for the downward shift of the LCST, allowing the polymer to push out water at lower temperatures.

Furthermore, the swollen and collapsed state were characterized by plotting the swelling ratio (measured thickness normalized by the respective thickness measured in dry $\mathrm{N}_{2}$ atmosphere) in the swollen (@20 ${ }^{\circ} \mathrm{C}$ ) as well as in the collapsed state in Figure 3b. ${ }^{a}$ Therefore, the more hydrophobic nature of the thin films with higher amount of cross-linking can be seen in the degree of swelling at $20^{\circ} \mathrm{C}$ (swollen) decreasing with the DEGDVE fraction. Please note, that for the more cross-linked samples $20{ }^{\circ} \mathrm{C}$ is also closer to the LCST (cf. Figure 3a). However, in the collapsed state, the thickness normalized by the dry thickness also decreases with the DEGDVE content of the thin films (see Figure $3 b$ ). This can be attributed to the fact that the mesh size of the more crosslinked thin films is smaller and, therefore, less water is retained in the polymeric system in the collapsed state.

Wettability. As the WCA probes a sample in terms of wettability and surface energy, the temperature-dependent swelling behavior of the differently cross-linked p(NVCL-co-
DEGDVE) thin-film samples was also investigated by WCA measurements (Figure 4). To avoid the kinetic effect of
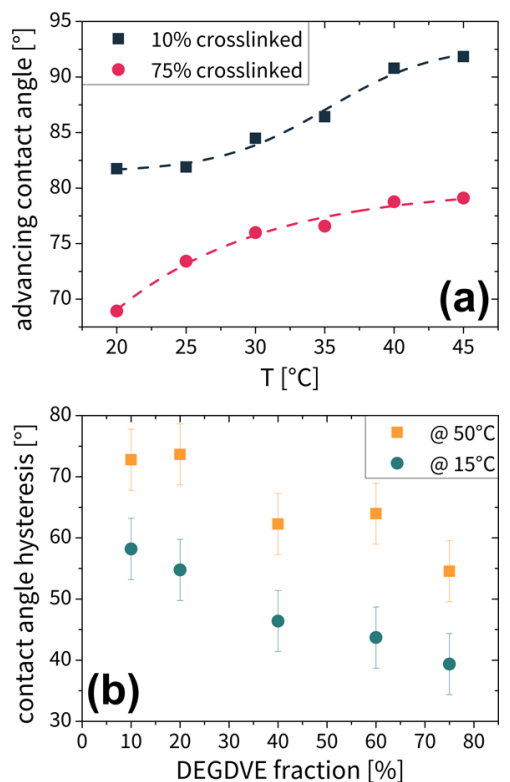

Figure 4. (a) Advancing WCA of two differently cross-linked $\mathrm{p}$ (NVCL-co-DEGDVE) thin-film samples measured as a function of substrate temperature (dotted lines are for guidance of the eye; error bars are $\pm 5^{\circ}$ but have not been included in the plot for clarity) and (b) WCA hysteresis as a function of DEGDVE fraction for various differently cross-linked $\mathrm{p}$ (NVCL-co-DEGDVE) samples measured at 15 and $50{ }^{\circ} \mathrm{C}$.

swelling interfering with the measurement, advancing and receding WCAs were determined. The advancing WCAs measured at several temperatures around the LCST transition of two differently cross-linked polymers (10 and 75\%) are plotted in Figure 4a. The advancing WCAs of the more crosslinked samples are lower than the values of the less cross-linked ones for all the substrate temperatures recorded. As the advancing WCA is viewed as a probe of the dry surface, these measurements hint at a more hydrophobic nature of the dry surface for the less cross-linked films. This can probably be attributed to the higher chain mobility compared to the more cross-linked sample, resulting in more surface rearrangement and more hydrophobic groups getting exposed to the polymer-air interface. The SE data showed that the less cross-linked samples take up significantly more water in the whole range of investigated temperatures (cf. Figure 2). This is in agreement with the receding angle of the less cross-linked sample (10\%) being lower than the receding angle of the more cross-linked one (75\%). The corresponding receding WCAs are temperature-independent, exhibiting mean values of $(21 \pm$ $5)^{\circ}$ and $(29 \pm 5)^{\circ}$, respectively. The ellipsometric data of Figure 2 are taken in water and, therefore, they should be compared to the WCA measured after surface rearrangement at the polymer-water interface, that is, the receding angle. In addition, while SE records the consequences of the LCST transition averaged over the whole layer in depth (as film thickness and refractive index), WCA measurements are very surface-sensitive, probing only the outermost surface of the films. Therefore, SE shows a higher amount of water taken up by a less cross-linked layer with the surface being more hydrophilic as evaluated from receding WCA measurements. 
Moreover, the advancing WCA results (Figure 4a) give evidence of a temperature-dependent transition of the surface from more hydrophilic at lower temperatures to more hydrophobic at higher temperatures. These results in temperature-dependent hydrophobicity/philicity of the surface also show the difference in transition temperature for the differently cross-linked samples, similar to the LCST values gained from SE measurements (cf. Figure 3a).

Furthermore, WCA measurements reveal that the WCA hysteresis decreases with increasing the amount of crosslinking for the investigated series of $\mathrm{p}$ (NVCL-co-DEGDVE) thin-film samples (see Figure 4b). The WCA hysteresis, as the difference between the advancing and receding WCAs, gives a measure about the ability of the surface for restructuring during water exposure. Hence, it is clear that cross-linking reduces this "flexibility" of the (surface) structure. Moreover, at higher temperatures, the more hydrophobic nature of the surface (in water) also makes for a higher hysteresis.

Mechanical Properties. To gain further understanding on the polymeric structures in the aqueous environment, the mechanical properties of the films have been probed by in situ force curve measurements. Force-separation profiles for the differently cross-linked samples were measured in water at 25 ${ }^{\circ} \mathrm{C}$ (Figure 5a). Every measurement can be understood as the
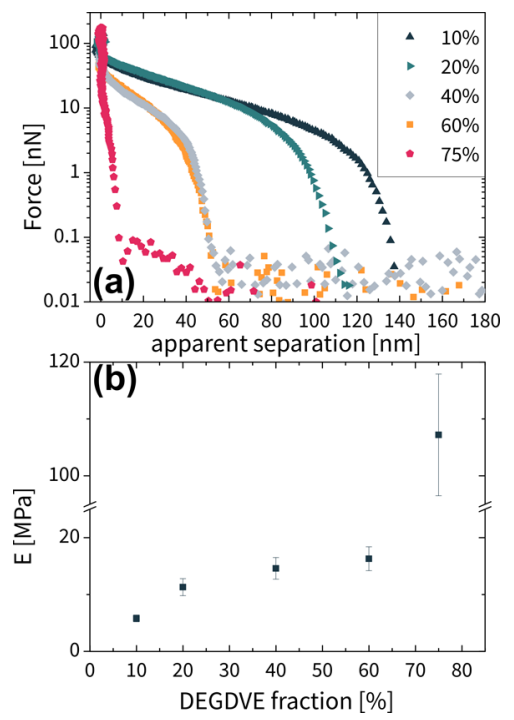

Figure 5. Exemplary force-separation curves as measured by AFM for differently cross-linked $\mathrm{p}$ (NVCL-co-DEGDVE) samples (a) and their respective apparent elastic modulus $E$, as determined from the Hertz equation (b). The measurements were taken in water at $25^{\circ} \mathrm{C}$.

tip of the cantilever of the atomic force microscope (AFM) advancing toward the substrate surface from top (high apparent separation). Eventually, the tip gets in contact and starts to penetrate into the polymeric mesh (when the measured force starts to be larger than the noise). Upon further movement, the repulsion and the measured deflectional force increase and reach maximum values at $0 \mathrm{~nm}$ apparent separation; at this point, the mechanical properties of the cantilever used do not allow any further penetration. All the measured curves exhibit the described behavior; more crosslinked samples are less compressible than the ones with the lower cross-linker amount. The point where the tip starts to penetrate into the polymer mesh can be compared to the film thickness measured by $\mathrm{SE}$ at the respective temperature (cf.
Figure 2). For the least cross-linked samples (10 and 20\%), the corresponding apparent separation is about $10-20 \mathrm{~nm}$ lower than the measured film thickness. Thus, the tip can penetrate most of the polymeric layer because of the mesh being large and/or the polymer being elastic enough. It should be noted that, in general, the true separation of the AFM tip from the substrate surface cannot be estimated using a setup like ours; the measured separation values just reflect the distance the tip is capable of compressing/penetrating the polymeric layer until the mechanical resistance of the layer gets too high. For the more cross-linked samples, the difference between the maximal apparent compression and the results from SE becomes more significant, meaning that the higher cross-linking results in a stronger resistance against the indentation of the tip, or its compression. For the sample with $75 \%$ cross-linking, hardly any compression can be observed, even though the sample has a nominal layer thickness of $\sim 65 \mathrm{~nm}$ in water at $25^{\circ} \mathrm{C}$. In fact, at this temperature, the more cross-linked samples are closer to or even above their LCST (cf. Figure 3a). The 75\% crosslinked sample is, hence, in its "collapsed state", hypothetically adding to the high stiffness observed (cf. Figure $5 \mathrm{~b}$ ).

By modeling the data, the apparent elastic modulus $(E)$ of the deposited systems could be extracted. To describe the force $(F)$ as a function of the indentation depth $(\delta)$, we applied the Hertz equation for a parabolic shape AFM tip: ${ }^{24}$

$$
F=\frac{4 \sqrt{r_{\text {tip }}}}{3\left(1-\nu^{2}\right)} E \delta^{3 / 2}
$$

As given by the manufacturer, the radius of the tip $\left(r_{\text {tip }}\right)$ is 10 $\mathrm{nm}$. The Poisson ratio $\nu$ was assumed as 0.5 and the spring constant of the cantilever used for all measurements was measured to be $0.24 \mathrm{~N} / \mathrm{m}$. The $E$ values for the various samples are summarized in Figure $5 \mathrm{~b}$. $E$ increases from 5.8 $\mathrm{MPa}$ for $10 \%$ to $107.2 \mathrm{MPa}$ for $75 \%$ nominal cross-linking measured at $25{ }^{\circ} \mathrm{C}$ in water, showing that with cross-linking, it is also possible to tune the mechanical properties. In comparison, for various end-grafted and highly extended polymer brushes, $E$ values between 0.2 and $0.3 \mathrm{MPa}$ have been reported, ${ }^{24}$ while polymer brushes with 10 times higher grafting density show values in the range $30-80 \mathrm{MPa}^{25}$ Furthermore, pNVCL has been previously employed to increase the elastic modulus of silicone rubber films from 1 $\mathrm{MPa}$ up to above $100 \mathrm{MPa}{ }^{26}$ In the present study, more crosslinking allowed for reaching high values of $E$ in pNVCL-based thin films at a given temperature; this can be advantageous in actuator setups, where higher stiffness is in demand.

To investigate how the elasticity changes as the swelling is responding to a change in temperature, exemplary force curves for the sample of $10 \%$ cross-linking at different temperatures above and below its LCST of $\sim 40{ }^{\circ} \mathrm{C}$ are shown in Figure 6 . At low temperatures, the layer can be compressed by about $140 \mathrm{~nm}$. Increasing the temperature leads to a significant reduction of compression because of the collapse of the mesh. The calculation of the apparent elasticity reveals that the initial value of $E$ at $25^{\circ} \mathrm{C}(5.8 \mathrm{MPa})$ increases to $8.4 \mathrm{MPa}$ at $36^{\circ} \mathrm{C}$ and $12.2 \mathrm{MPa}$ at $46^{\circ} \mathrm{C}$. The lower amount of swelling and the corresponding increased repulsion of adjacent meshes results in a reduction of the flexibility upon indentation.

Filament Temperature and Thermoresponsiveness. To investigate the effect of molecular weight on the LCST transition of $\mathrm{p}$ (NVCL-co-DEGDVE) thin films, the filament temperature $\left(T_{\text {fil }}\right)$ was used to control the concentration of 


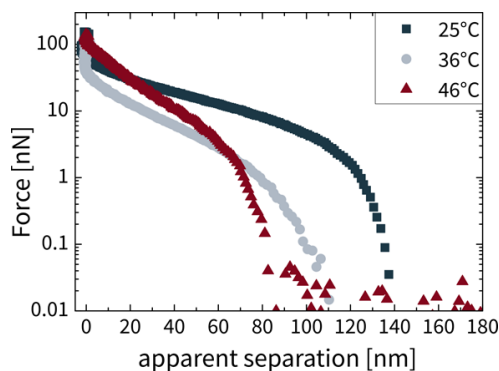

Figure 6. Force-separation curves as measured by AFM on a $10 \%$ cross-linked $\mathrm{p}$ (NVCL-co-DEGDVE) sample at various temperatures above and below the LCST of $\sim 40{ }^{\circ} \mathrm{C}$ in water.

initiator radicals during polymerization. At $\sim 150{ }^{\circ} \mathrm{C}, T_{\text {fil }}$ is sufficient to break the peroxide bond in the initiator molecules, leading to the formation radicals that initiate polymerization. Above $150{ }^{\circ} \mathrm{C}$, Ozaydin-Ince et al. showed that the deposition rate increases proportionally to $T_{\text {fil }}$ i hence, the filament temperature is proportional to the concentration of initiator radicals formed. ${ }^{27}$ This relation makes it possible to distinguish between the initiation and the propagation step during polymerization, as only the first depends on the concentration of initiator radicals. ${ }^{16}$ A lower filament temperature causes fewer radicals to be formed; while the initiation of new polymer chains should be decreased, the propagation of chains already existing remains unchanged, as this process does not require the presence of initiator radicals. Besides that, fewer termination events of polymer chains by initiator radicals will occur. Thus, the resulting smart polymer thin film should exhibit a polymeric structure with a larger molecular weight (i.e., longer chains). From the literature, it is known that for low-filament temperatures $\left(<270{ }^{\circ} \mathrm{C}\right)$, TBPO dominantly decomposes by cleavage of the peroxide bond; in this case, primarily, the tert-butoxy radical is formed. ${ }^{27}$ To ensure this (for reasons of simplicity), a series of samples has been deposited at filament temperatures between 165 and $215{ }^{\circ} \mathrm{C}$ with a constant nominal cross-linker amount of $20 \%$ with and without a patch flow of $\mathrm{N}_{2}$. The flow rates of monomer, crosslinker, and initiator remained unchanged throughout the series.

The low amounts (nm film thickness) of material deposited and the low solubility in common solvents due to cross-linking, make the experimental determination of the molecular weight not possible. However, the thermoresponsive swelling curves for samples deposited with $\mathrm{N}_{2}$ (see Figure 7a) indicate a strong difference in response behavior depending on the filament temperature in terms of transition temperature. The LCST decreases by $16{ }^{\circ} \mathrm{C}$ when $T_{\text {fil }}$ is reduced from 200 down to 165 ${ }^{\circ} \mathrm{C}$. Such change in LCST is twice as large as the one obtained by Meeussen et al. when the molecular weight of a pNVCL homopolymer was increased from 9 to $275 \mathrm{~kg} / \mathrm{mol}{ }^{14}$ This behavior can be attributed to the presence of DEGDVE as a cross-linking agent, being reported to lower the transition temperature also in NIPAAm-based co-polymers. ${ }^{15}$

Interestingly, the degrees of swelling of these samples (nominally $20 \%$ cross-linked, with $\mathrm{N}_{2}$ patch flow) are more comparable to the one of the nominally $10 \%$ cross-linked sample of the previous cross-linker series (Figure $7 \mathrm{~b}$ ). However, the LCST at $\sim 33{ }^{\circ} \mathrm{C}$ still remains in the range of the nominally $20 \%$ cross-linked sample of that very series. Here, it has to be noted that introducing nitrogen as a carrier gas also reduces the $p_{\mathrm{M}} / p_{\text {sat }}$ values of the monomer and crosslinker used during iCVD. For example, deposition at a filament

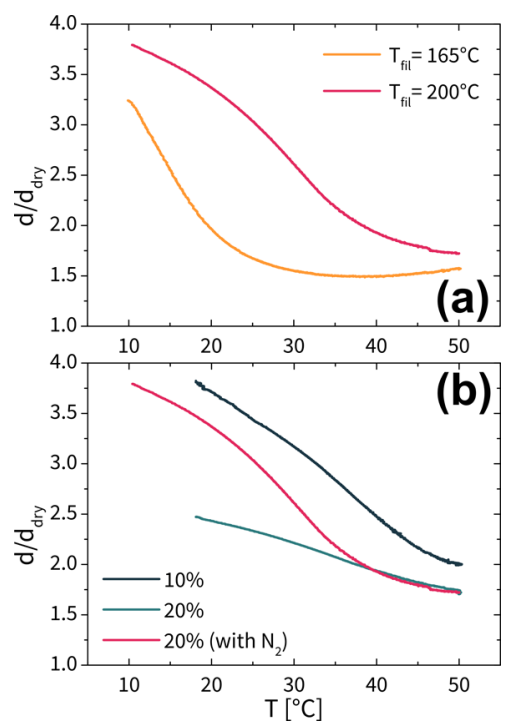

Figure 7. Thickness of the swollen layers normalized by the dry thickness $d / d_{\text {dry }}$ recorded by SE in water, while applying heating ramps from $\sim 10$ to $50{ }^{\circ} \mathrm{C}$, for two nominally $20 \%$ cross-linked $\mathrm{p}$ (NVCL-co-DEGDVE) thin-film samples deposited (a) at different filament temperatures $\left(T_{\text {fil }}\right)$ with $\mathrm{N}_{2}$ patch flow; (b) measurement curves of samples deposited at $T_{\text {fil }}=200{ }^{\circ} \mathrm{C}$ without $\mathrm{N}_{2}$ with nominal cross-linking of 10 and $20 \%$ (cf. Figure 2) plotted together with the respective sample deposited with $\mathrm{N}_{2}$ [see. (a)] for comparison of the degrees of swelling.

temperature of $200{ }^{\circ} \mathrm{C}$ and $2 \mathrm{sccm} \mathrm{N}$ patch flow results in an average growth rate of $0.7 \mathrm{~nm} / \mathrm{min}$; lowering $T_{\text {fil }}$ to $165{ }^{\circ} \mathrm{C}$ even yields $0.1 \mathrm{~nm} / \mathrm{min}$. Furthermore, a comprehensive review of pNVCL-based polymers showed that polymerization kinetics are challenging and controlling the co-polymerization of NVCL with other monomers is difficult because of the low vapor pressure of the NVCL monomer. ${ }^{13}$ Growth kinetics seem to play an important role also in the co-polymerization process of NVCL with DEGDVE, but will be addressed in a future work, as this would go beyond the scope of the current contribution. Hypothetically, with changing the $p_{\mathrm{M}} / p_{\text {sat }}$ the propagation kinetics are altered for NVCL and DEGDVE in a different way. The slower growth at lower $p_{\mathrm{M}} / p_{\text {sat }}$ (i.e., $\mathrm{N}_{2}$ carrier gas) appears to alter the polymeric structure toward a more open mesh only being apparent when swollen. As the depositions are run in dry conditions (in vacuum), the polymer mesh grows in a collapsed state (as a reference state, cf. Harmon et al. $\left.{ }^{28}\right)$. Hence, also in water, the thickness fraction at elevated temperatures (i.e., collapsed state) is comparable to the one of the $20 \%$ cross-linked sample deposited without $\mathrm{N}_{2}$ patch flow (see Figure $7 \mathrm{~b}$ ).

Nevertheless, with $\mathrm{N}_{2}$ patch flow, by lowering the filament temperature from 200 to $165^{\circ} \mathrm{C}$, the degree of swelling stays comparable, whereas at the same time, the transition temperature decreases significantly, to $\sim 16{ }^{\circ} \mathrm{C}$. The longer polymer chains (i.e., lower filament temperature) appear to result in a similar mesh size, but a larger overall cross-linked structure making it possible to repel out water at lower temperatures. In the described way, the response of the newly developed thermoresponsive polymeric thin films can be tuned in transition temperature and toward very high degrees of swelling. Together with the resulting sharper transition (cf. Figure 7), this tunability is crucial for the application in smart sensor setups. 
A similar series of samples, deposited at different $T_{\text {fil }}$ but without $\mathrm{N}_{2}$, also resulted in sharper transitions for samples deposited at lower filament temperature, but constant transition temperature (see Supporting Information). The reason for such a behavior (e.g., unchanged molecular weight) could be the fact that the used working conditions result in a mass transfer-limited process during iCVD synthesis of the polymer thin films. In these conditions, it has been reported that the filament temperature does not affect the deposition as it does in the reaction-kinetic regime (i.e., at higher flow rates). ${ }^{27}$

\section{CONCLUSIONS}

Novel pNVCL-based co-polymer thin films exhibiting promising thermoresponsive properties for sensor and actuator setups have been synthesized via iCVD for the first time. This study sheds light onto the swelling behavior of these $\mathrm{p}$ (NVCLco-DEGDVE) systems and aims to reveal connections between deposition parameters, material properties, and the thermoresponsiveness of the deposited polymeric structures. Stable hydrogel thin films with different amounts of cross-linking were synthesized. The cross-linker amount was shown to change the amount of water uptake, with less cross-linked samples exhibiting higher swelling ratios. However, samples exhibiting nominal cross-linking below $10 \%$ are not stable in water. Furthermore, with increasing the amount of crosslinking, the transition temperature was shown to decrease because of the hydrophobicity of the cross-linker used.

The LCST transition has been observed to be present as a transition in wettability in WCA experiments. Furthermore, the WCA hysteresis showed that the surfaces are keener to rearrange in less cross-linked polymers. A force-separation study on the mechanical properties of the thin films by an AFM tip also revealed a denser polymeric mesh for more crosslinked samples with the apparent elastic modulus changing as a function of the cross-linker amount from 5.8 to 107.2 MPa at a constant temperature of $25{ }^{\circ} \mathrm{C}$. During deswelling from temperatures below to above the LCST, the apparent elastic modulus of a $10 \%$ cross-linked sample is observed to double.

Moreover, the filament temperature affecting the chain length of the polymer has successfully been used to alter the thermoresponsiveness of $\mathrm{p}$ (NVCL-co-DEGDVE) thin films in a large range of transition temperatures $\left(16-33^{\circ} \mathrm{C}\right.$ for similar cross-linking). Further investigations on the complex growth kinetics that lead to changes in the swelling behavior need to be addressed in future contributions.

Overall, we report on the successful synthesis of polymer thin films that swell up to $250 \%$ when compared to their thickness in the dry state $\left(\mathrm{N}_{2}\right.$ environment) with tunable transition temperatures in the range $16-40{ }^{\circ} \mathrm{C}$, which is interesting for various applications in sensor and actuator setups.

\section{ASSOCIATED CONTENT}

\section{S Supporting Information}

The Supporting Information is available free of charge on the ACS Publications website at DOI: 10.1021/acs.macromol.9b01364.

Thickness and index as a function of temperature of a $40 \%$ cross-linked p(NVCL-co-DEGDVE) thin-film sample with their respective first and second derivatives to exemplarily show the evaluation of the LCST value from the data and swelling as a function of temperature in water of various $20 \%$ cross-linked p(NVCL-coDEGDVE) thin-film samples deposited at filament temperatures between 185 and $215{ }^{\circ} \mathrm{C}$ without $\mathrm{N}_{2}$ patch flow (PDF)

\section{AUTHOR INFORMATION}

\section{Corresponding Author}

*E-mail: anna.coclite@tugraz.at. ORCID

Fabian Muralter: 0000-0001-8038-6019

Alberto Perrotta: 0000-0002-7007-6153

Oliver Werzer: 0000-0003-0732-4422

Anna Maria Coclite: 0000-0001-5562-9744

Notes

The authors declare no competing financial interest.

\section{ACKNOWLEDGMENTS}

This project has received funding from the European Research Council (ERC) under the European Union's Horizon 2020 research and innovation program (grant agreement 715403).

\section{ADDITIONAL NOTE}

${ }^{a}$ The collapsed state has been defined as the point, where the thickness is minimal from the curves recorded during temperature-dependent de-swelling upon heating (cf. Figure $\left.{ }^{2,}\right)$. For the samples with 10 and $20 \%$ nominal DEGDVE fraction, the values at $50{ }^{\circ} \mathrm{C}$ have been reported, as it is the upper limit of the measurement setup, although the LCSTrelated de-swelling process is apparently not entirely finished at $50{ }^{\circ} \mathrm{C}$ in these cases (cf. respective curves in Figure ${ }^{2}$ ). In the collapsed state, for the more cross-linked samples, an increase in film thickness was observed after full de-swelling. We think that this can be attributed to thermal expansion of the polymer in water; this will be discussed in a following contribution.

\section{REFERENCES}

(1) Palagi, S.; Mark, A. G.; Reigh, S. Y.; Melde, K.; Qiu, T.; Zeng, H.; Parmeggiani, C.; Martella, D.; Sanchez-Castillo, A.; Kapernaum, N. Structured Light Enables Biomimetic Swimming and Versatile Locomotion of Photoresponsive Soft Microrobots. Nat. Mater. 2016, $15,647-653$.

(2) Ramakrishnan, N.; Vamsi, T.; Khan, A.; Nemade, H. B.; Palathinkal, R. P. Humidity Sensor Using NIPAAm Nanogel as Sensing Medium in SAW Devices. Int. J. Nanosci. 2011, 10, 259-262.

(3) Chu, L. Y.; Xie, R.; Ju, X. J.; Wang, W. Smart Hydrogel Functional Materials. Smart Hydrogel Functional Materials; SpringerVerlag: Berlin, Heidelberg, 2013; pp V-XVIII.

(4) Beebe, D. J.; Moore, J. S.; Bauer, J. M.; Yu, Q.; Liu, R. H.; Devadoss, C.; Jo, B.-H. Functional Hydrogel Structures for Autonomous Flow Control inside Micro-Fluidic Channels. Nature 2000, 404, 588-590.

(5) Canal, T.; Peppas, N. A. Correlation between Mesh Size and Equilibrium Degree of Swelling of Polymeric Networks. J. Biomed. Mater. Res. 1989, 23, 1183-1193.

(6) Lau, K. K. S.; Gleason, K. K. Initiated Chemical Vapor Deposition (ICVD) of Poly (Alkyl Acrylates): An Experimental Study. Macromolecules 2006, 39, 3688-3694.

(7) Liu, R.; Fraylich, M.; Saunders, B. R. Thermoresponsive Copolymers: From Fundamental Studies to Applications. Colloid Polym. Sci. 2009, 287, 627-643.

(8) Panayiotou, M.; Freitag, R. Influence of the Synthesis Conditions and Ionic Additives on the Swelling Behaviour of Thermo-Responsive Polyalkylacrylamide Hydrogels. Polymer 2005, 46, 6777-6785. 
(9) Salzmann, P.; Perrotta, A.; Coclite, A. M. Different Response Kinetics to Temperature and Water Vapor of Acrylamide Polymers Obtained by Initiated Chemical Vapor Deposition. ACS Appl. Mater. Interfaces 2018, 10, 6636-6645.

(10) Alf, M. E.; Hatton, T. A.; Gleason, K. K. Novel NIsopropylacrylamide Based Polymer Architecture for Faster LCST Transition Kinetics. Polymer 2011, 52, 4429-4434.

(11) Pena-Francesch, A.; Montero, L.; Borrós, S. Tailoring the LCST of Thermosensitive Hydrogel Thin Films Deposited by ICVD. Langmuir 2014, 30, 7162-7167.

(12) Lee, B.; Jiao, A.; Yu, S.; You, J. B.; Kim, D.-H.; Im, S. G. Initiated Chemical Vapor Deposition of Thermoresponsive Poly (NVinylcaprolactam) Thin Films for Cell Sheet Engineering. Acta Biomater. 2013, 9, 7691-7698.

(13) Cortez-Lemus, N. A.; Licea-Claverie, A. Poly(N-Vinylcaprolactam), a Comprehensive Review on a Thermoresponsive Polymer Becoming Popular. Prog. Polym. Sci. 2016, 53, 1-51.

(14) Meeussen, F.; Nies, E.; Berghmans, H.; Verbrugghe, S.; Goethals, E.; Du Prez, F. Phase Behaviour of Poly ( N - Vinyl Caprolactam ) in Water. Polymer 2000, 41, 8597-8602.

(15) Muralter, F.; Perrotta, A.; Coclite, A. M. Thickness-Dependent Swelling Behavior of Vapor-Deposited Smart Polymer Thin Films. Macromolecules 2018, 51, 9692-9699.

(16) Lau, K. K. S.; Gleason, K. K. Initiated Chemical Vapor Deposition (ICVD) of Poly(Alkyl Acrylates): A Kinetic Model. Macromolecules 2006, 39, 3695-3703.

(17) Christian, P.; Coclite, A. M. Vapor-Phase-Synthesized Fluoroacrylate Polymer Thin Films: Thermal Stability and Structural Properties. Beilstein J. Nanotechnol. 2017, 8, 933-942.

(18) Sader, J. E.; Chon, J. W. M.; Mulvaney, P. Calibration of Rectangular Atomic Force Microscope Cantilevers. Rev. Sci. Instrum. 1999, 70, 3967-3969.

(19) Butt, H.-J.; Cappella, B.; Kappl, M. Force Measurements with the Atomic Force Microscope: Technique, Interpretation and Applications. Surf. Sci. Rep. 2005, 59, 1-152.

(20) Hermanowicz, P.; Sarna, M.; Burda, K.; Gabryś, H. AtomicJ: An Open Source Software for Analysis of Force Curves. Rev. Sci. Instrum. 2014, 85, 063703.

(21) Coclite, A. M.; Lund, P.; Di Mundo, R.; Palumbo, F. Novel Hybrid Fluoro-Carboxylated Copolymers Deposited by Initiated Chemical Vapor Deposition as Protonic Membranes. Polymer (Guildf) 2013, 54, 24-30.

(22) Alf, M. E.; Hatton, T. A.; Gleason, K. K. Initiated Chemical Vapor Deposition of Responsive Polymeric Surfaces. Thin Solid Films 2011, 519, 4412-4414.

(23) Lau, K. K. S.; Gleason, K. K. Initiated Chemical Vapor Deposition (ICVD) of Poly(Alkyl Acrylates): An Experimental Study. Macromolecules 2006, 39, 3688-3694.

(24) Kutnyanszky, E.; Vancso, G. J. Nanomechanical Properties of Polymer Brushes by Colloidal AFM Probes. Eur. Polym. J. 2012, 48, $8-15$.

(25) Julthongpiput, D.; LeMieux, M.; Tsukruk, V. V. Micromechanical Properties of Glassy and Rubbery Polymer Brush Layers as Probed by Atomic Force Microscopy. Polymer 2003, 44, 45574562.

(26) Pino-Ramos, V. H.; Flores-Rojas, G. G.; Alvarez-Lorenzo, C.; Concheiro, A.; Bucio, E. Graft Copolymerization by Ionization Radiation, Characterization, and Enzymatic Activity of TemperatureResponsive SR-g-PNVCL Loaded with Lysozyme. React. Funct. Polym. 2018, 126, 74-82.

(27) Ozaydin-Ince, G.; Gleason, K. K. Transition between Kinetic and Mass Transfer Regimes in the Initiated Chemical Vapor Deposition from Ethylene Glycol Diacrylate. J. Vac. Sci. Technol., A 2009, 27, 1135-1143.

(28) Harmon, M. E.; Kuckling, D.; Pareek, P.; Frank, C. W. PhotoCross-Linkable PNIPAAm Copolymers. 4. Effects of Copolymerization and Cross-Linking on the Volume-Phase Transition in Constrained Hydrogel Layers. Langmuir 2003, 19, 10947-10956. 